\title{
A modified HPLC method improves the simultaneous determination of plasma kynurenine and tryptophan concentrations in patients following maintenance hemodialysis
}

\author{
CHENGGEN XIAO ${ }^{1,2 *}$, YUANHAN CHEN $^{2 *}$, XINLING LIANG $^{1,2}, \mathrm{ZHEN} \mathrm{XIE}^{3}$, MIN ZHANG $^{4}$, \\ RUIZHAO LI ${ }^{2}$, ZHILIAN LI ${ }^{1,2}$, XIA FU ${ }^{1,2}$, XIYONG YU ${ }^{5}$ and WEI SHI ${ }^{2}$ \\ ${ }^{1}$ Nanfang Medical University, Guangzhou, Guandong 510080; ${ }^{2}$ Division of Nephrology, Guangdong General Hospital, \\ Guangdong Academy of Medical Sciences, Guangzhou, Guandong 510080; ${ }^{3}$ Department of Dermatology, \\ Sichuan Academy of Medical Sciences and Sichuan Provincial People's Hospital, Chengdu, Sichuan 610000; \\ ${ }^{4}$ Department of Gastroenterology, The Sixth Affiliated Hospital, Sun Yat-Sen University; ${ }^{5}$ Medical Research Center, \\ Guangdong General Hospital, Guangdong Academy of Medical Sciences, Guangzhou, Guangdong 510081, P.R. China
}

Received September 2, 2013; Accepted January 14, 2014

DOI: 10.3892/etm.2014.1512

\begin{abstract}
The ratio between plasma kynurenine (Kyn) and tryptophan (Trp) serves as a marker of indoleamine 2,3-dioxygenase, a critical immunomodulatory molecule. Simultaneous detection of the two markers may be performed using high-pressure liquid chromatography (HPLC). However, for uremic patients, the conventional detection method may be affected by a range of accumulated toxins. The current study aimed to establish a method for the simultaneous measurement of Kyn and Trp in patients following maintenance hemodialysis via HPLC-ultraviolet detection. The procedure involved the use of a SinoChrom ODS-BP C18 column (4.6x150 mm; inner diameter, $4.5 \mu \mathrm{m})$ and a mobile phase of $15 \mathrm{mmol} / \mathrm{l}$ sodium acetate acetic acid solution (containing 5\% acetonitrile, $\mathrm{pH}$ 4.8). The modified method was verified using plasma samples from 10 healthy controls and 91 maintenance hemodialysis patients. The results demonstrated that the modified method was successful in simultaneously detecting the concentrations of Trp and Kyn in the healthy controls and maintenance hemodialysis patients. The method is simple, fast, accurate and suitable for clinical and research purposes in maintenance hemodialysis patients.
\end{abstract}

Correspondence to: Dr Xinling Liang, Division of Nephrology, Guangdong General Hospital, Guangdong Academy of Medical Sciences, 106 Zhongshan Road 2, Guangzhou, Guandong 510080, P.R. China

E-mail: liangxinlingen@163.com

${ }^{*}$ Contributed equally

Key words: high-pressure liquid chromatography, kynurenine, tryptophan, hemodialysis, immunological biomarker, indoleamine 2,3-dioxygenase

\section{Introduction}

End-stage renal disease seriously affects human health and maintenance hemodialysis is the primary therapy for this condition. Although the technology for maintenance hemodialysis is continually being developed, malnutrition and inflammation significantly affect patient prognosis (1). Previous studies have shown that tryptophan (Trp) is an important regulator of immune homeostasis and inflammation (2). Trp is one of the essential amino acids in the human body and has a critical role in effective $\mathrm{T}$ cell function. Depletion of Trp activates effector $\mathrm{T}$ lymphocytes, thereby mediating immune tolerance. Furthermore, a metabolite of Trp, kynurenine (Kyn), promotes the generation of regulatory $\mathrm{T}$ lymphocytes, which further promote immune tolerance (3). Indoleamine 2,3-dioxygenase (IDO) is an intercellular rate-limiting enzyme that catalyzes the conversion of Trp to Kyn and plays a key role in immune homeostasis in uremic patients (2,4-6). IDO is also an immunoregulatory signal transduction molecule (7). Therefore, Kyn functions as a new potential marker in patients with end-stage renal disease.

IDO activity can be estimated by the concentration ratio of Kyn to Trp $(5,8)$. The Kyn/Trp ratio correlates with inflammatory markers, including high-sensitivity C-reactive protein and soluble tumor necrosis factor-receptor-1, and has been recognized as a promising biomarker for evaluating immune homeostasis and inflammation $(4,5)$. High-pressure liquid chromatography (HPLC) is a traditional method for measuring plasma amino acids levels. However, simultaneous measurement of Trp and Kyn is difficult using HPLC (9). Schefold et al reported a costly electrospray-tandem mass spectrometry assay for measuring Trp and Kyn simultaneously (5). With regard to clinical practice, several previous approaches to simultaneously measuring Trp and Kyn using HPLC have been reported $(10,11)$. However, these methods were limited to isolating Kyn in uremic patients due to an interference of unknown uremic toxin (Fig. 1A). Additional HPLC methods have been used for uremic patients, but 
these methods have tedious and time-consuming procedures $(12,13)$.

The present study aimed to develop a modified method to measure Trp and Kyn simultaneously in uremic patients for the evaluation of IDO activity.

\section{Materials and methods}

Reference method. The conventional method for simultaneous Trp and Kyn detection was used as the reference. A SymmetryShield RP-C18 column (150x3.9 mm; inner diameter, $5 \mu \mathrm{m}$ ) was purchased from Waters Corporation (Milford, MA, USA). The column had a mobile phase of $2.7 \%$ acetonitrile and $\mathrm{pH} 3.6$ (11).

Instruments. An LC-3A high-pressure liquid chromatograph (Shimadzu, Kyoto, Japan), SCI 100 UV-Vis detector (Daniel Elite Analytical Industries, Inc., Houston, TX, USA), YH-300 data processing system (Easeatech, Guangzhou, China) and VICILC 25- $\mu 1$ manual injector (Shimadzu, Kyoto, Japan) were used in the procedure.

Reagents. Standard samples of Kyn and L-Trp were purchased from Sigma-Aldrich (St. Louis, MO, USA). Chromatographic pure acetonitrile, potassium dihydrogen phosphate, sodium acetate, analytical-grade acetic acid, $36 \%$ perchloric acid and ultra-pure water were treated with a Milli-Q Pure water device (Millipore Corporation, Billerica, MA, USA).

Protein precipitant preparation. Perchloric acid solution at a volume fraction of $5.0 \%$ was prepared using ultra-pure water.

Mobile phase. Acetic acid (36\%) and acetonitrile solutions were used in the mobile phase. Sodium acetate crystals were weighed and sodium acetate-acetic acid solution (containing $5 \%$ acetonitrile) was prepared. Prior to use, the solution was ultrasonically degassed for $40 \mathrm{~min}$, incubated overnight and filtered using a precolumn (KJO-4282; Phenomenex, Torrance, CA, USA).

Preparation of the stock solution. Trp and Kyn stock solutions, at a concentration of $20 \mathrm{mmol} / \mathrm{l}$, were prepared using $2.5 \%$ perchloric acid solution. Aliquotted stock solutions were then stored at $-4^{\circ} \mathrm{C}$. Standard working solutions were prepared using ultra-pure water prior to each use.

Sample collection and processing. In total, 91 patients that were undergoing hemodialysis three times a week (single pool $\mathrm{Kt} / \mathrm{V},>1.2$ ) were recruited from the Blood Purification Center at Guangdong General Hospital (Guangzhou, China). An additional 10 healthy volunteers were also recruited (Table I). Blood samples $(2 \mathrm{ml})$ were collected from the arteriovenous fistulas of patients who had undergone maintenance hemodialysis and were placed into 4-ml EDTA tubes. After $1 \mathrm{~h}$, the blood was centrifuged at room temperature at 2,200 $\mathrm{x}$ g for $10 \mathrm{~min}$ to separate the plasma. Blood sample collection was approved by the Ethics Committee of Guangdong General Hospital $(2013069 H)$ and all patients provided informed consent.

Plasma samples $(50 \mu \mathrm{l})$ were placed into a centrifugal tube and the same volume of 5\% perchloric acid solution was
Table I. Characteristics of the control individuals and patients undergoing hemodialysis.

\begin{tabular}{lcc}
\hline Characteristics & Controls & Patients \\
\hline Age, years & $34 \pm 10$ & $53-77$ \\
Gender, male/female, $\mathrm{n}$ & $5 / 5$ & $51 / 40$ \\
Plasma Kyn concentration, $\mu \mathrm{mol} / \mathrm{l}$ & $0.8-0.4$ & $1.07 \pm 0.10$ \\
Plasma Trp concentration, $\mu \mathrm{mol} / 1$ & $31.3 \pm 21.2$ & $5.62 \pm 0.98$ \\
Plasma albumin, g/l & & $31.1 \pm 3.5$ \\
Hemodialysis time, days & & $1326.9 \pm 317.2$ \\
Single pool Kt/V* & & $1.5 \pm 0.3$ \\
\hline
\end{tabular}

Kyn, kynurenine; Trp, tryptophan.

added. Next, the samples were placed in a vortex mixer for $30 \mathrm{sec}$ and then maintained at room temperature for $15 \mathrm{~min}$ to allow plasma protein precipitation (11). Finally, the samples were centrifuged at $12,000 \mathrm{xg}$ for $5 \mathrm{~min}$ and $20-\mu 1$ samples of supernatant were collected for analysis.

Chromatographic requirements. The procedure involved a SinoChrom ODS-BP C18 column (4.6x150 mm; inner diameter, $4.5 \mu \mathrm{m}$; Dalian Elite Analytical Instruments Co., Ltd., Dalian, China) and a mobile phase of $15 \mathrm{mmol} / \mathrm{l}$ sodium acetate acetic acid solution (containing 5\% acetonitrile, $\mathrm{pH} 4.8$ ) at a flow rate of $1.0 \mathrm{ml} / \mathrm{min}$. The SC100-UV-visible detector was operated at $225 \mathrm{~nm}$ and the injection volume was $20 \mu \mathrm{l}$. Concentrations were measured at room temperature.

Preparation of mixed plasma. In total, 20 plasma samples were selected randomly from the 91 patients undergoing maintenance hemodialysis. The samples were mixed well and stored in a $-20^{\circ} \mathrm{C}$ freezer for later precision and recovery measurements (10).

Qualitative and quantitative analyses for Kyn and Trp. Qualitative analyses for Kyn and Trp were performed using the peak retention value comparison and superposition methods, while quantitative analysis was performed using the external standard method. Data processing was conducted using a YH-300 Chromatograph workstation (Easeatech). Quantitative results are expressed as mean \pm SD.

\section{Results}

Trp and Kyn concentrations. Concentrations of Trp and Kyn were detectable in all 10 healthy volunteers and in 90 of the 91 patients who underwent maintenance hemodialysis under the modified conditions (Table I). By contrast, the reference method only detected the concentrations of Trp and Kyn in 14 of the 91 patients undergoing maintenance hemodialysis. Failure of the reference method primarily arose from peak interference (Fig. 1A).

Standard curve and equation. Standard working solutions were prepared by combining $2.5 \%$ perchloric acid solution with Trp and Kyn (Kyn, 0.004-100 $\mu \mathrm{mol} / \mathrm{l}$; Trp, 0.01-1,000 $\mu \mathrm{mol} / \mathrm{l})$. 
Table II. Regression analysis and detection limits for Kyn and Trp.

\begin{tabular}{llccc}
\hline Substance & Regression equation & $\mathrm{R}^{2}$ & Linear range, $\mu$ mol/1 & Detection limit \\
\hline Kyn & $\mathrm{y}=28007 \mathrm{x}+3843.9$ & 1.0 & $0.08-50$ & 0.02 \\
Trp & $\mathrm{y}=27331 \mathrm{x}+85588$ & 1.0 & $0.8-500$ & 0.2 \\
\hline
\end{tabular}

$\mathrm{x}$, concentration $(\mu \mathrm{mol} / \mathrm{l}) . \mathrm{y}$, peak area ( $\mu \mathrm{V}$.sec). Kyn, kynurenine; Trp, tryptophan.

Table III. Precision of the intra- and inter-day measurements of Kyn and Trp $(n=20)$.

\begin{tabular}{lccc}
\hline Substance & Intra-day, mol/l & RSD, $\%$ & Inter-day, mol/l \\
\hline Kyn & $0.7301 \pm 0.0910$ & 2.60 & $0.7415 \pm 0.0250$ \\
Trp & $3.0280 \pm 0.0403$ & 1.33 & $2.9184 \pm 0.1279$ \\
\hline
\end{tabular}

Values are expressed as mean \pm SD. Kyn, kynurenine; Trp, tryptophan; RSD, relative standard deviation.

A

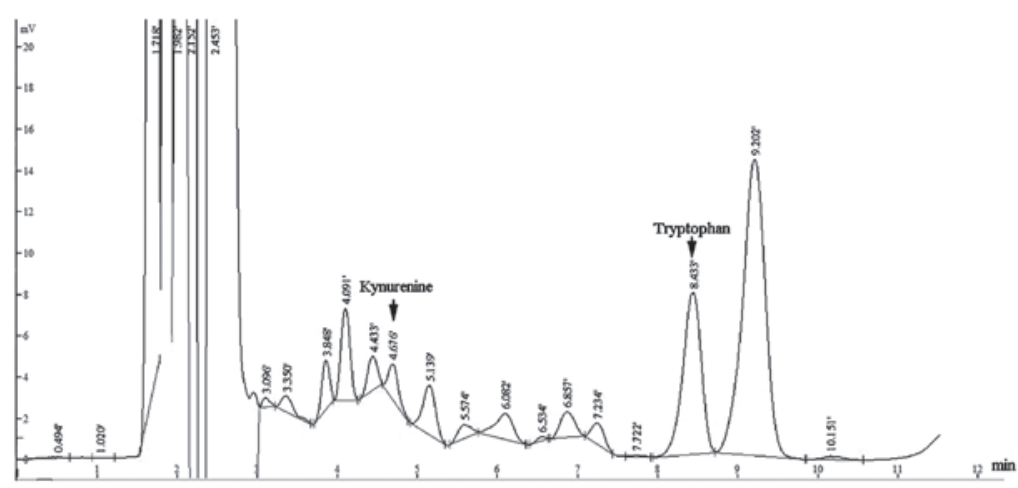

B

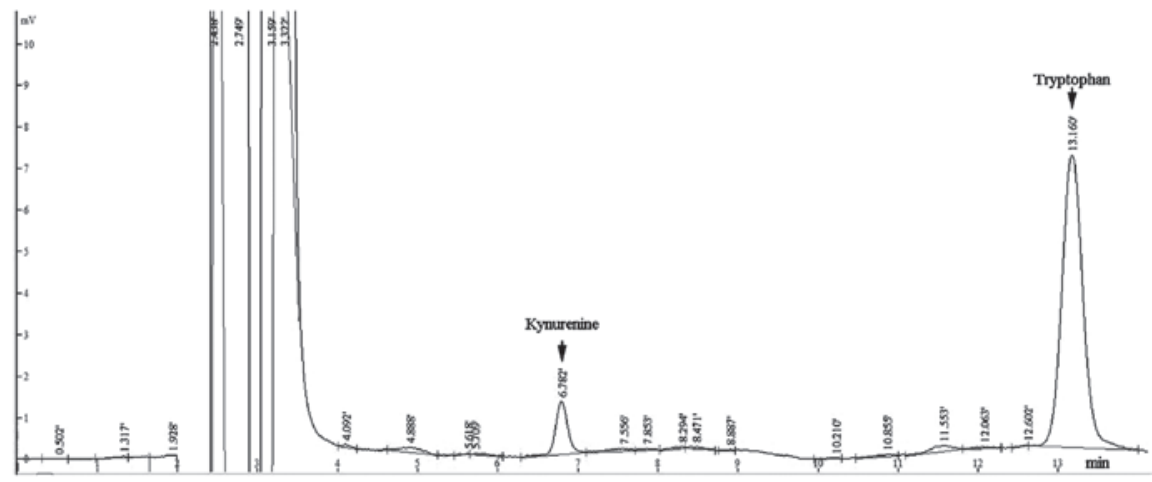

C

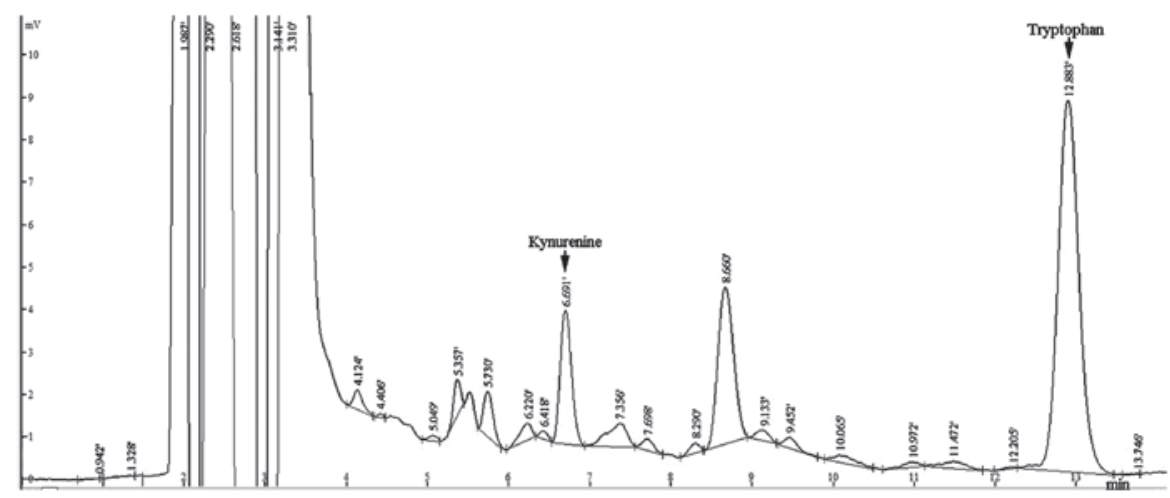

Figure 1. High-pressure chromatograms using the referenced and modified methods. Typical high-pressure chromatogram results using the (A) reference method for patients undergoing hemodialysis, (B) modified method for standard samples and (C) modified method for patients undergoing hemodialysis. Arrows indicate the peak positions of Trp and Kyn. Kyn, kynurenine; Trp, tryptophan. 
Each sample was injected three times and the average result was used for analysis. The results are shown in Table II, where $\mathrm{y}$ represents the peak area $(\mu \mathrm{V}$.sec $)$ and $\mathrm{x}$ is the sample concentration $(\mu \mathrm{mol} / \mathrm{l})$. A marked linear correlation was observed between the peak area and the sample concentrations of Kyn and Trp, with a wide linear range and a low detection limit, indicating good sensitivity.

Precision test. Chromatographic columns and precolumns were washed daily with formaldehyde and the standard curves were corrected every two days (10). Intra- and inter-day precision tests were performed on mixed plasma samples (Table III).

\section{Discussion}

$\mathrm{Kyn} / \mathrm{Trp}$ ratio is a marker that reflects immune homeostasis and inflammation. Although existing HPLC methods are able to rapidly and simultaneously detect Trp and Kyn in non-uremic patients, they fail to do so for uremic patients due to the presence of unknown uremic substances that have absorption profiles similar to that of Kyn (Fig. 1). In the present study, the column diameter, $\mathrm{pH}$ value of the mobile phase and the content of acetonitrile were adjusted to isolate Trp and Kyn from these interfering components (Fig. 1C). The results indicated that the modified method was able to simultaneously detect the concentrations of plasma Trp and Kyn in uremic patients.

The method used in the present study differs from previously reported HPLC methods. Zhen et al and Koening et al used a $5-\mu \mathrm{m}$ C18 column and adjusted the wavelength of the ultraviolet detector during detection $(12,13)$. In the current study, a 4.5- $\mu \mathrm{m} \mathrm{C18}$ column was used. Furthermore, the composition of the mobile phase was adjusted, which allowed for the detection of Kyn and Trp simultaneously at a fixed wavelength and with a shortened detection time. In addition, the current method has a wider detection range which completely covers the levels of human plasma Trp and Kyn under normal and pathological states. The modified method is also applicable for in vitro detection. However, in the present study, Trp and Kyn levels remained undetected in one sample. The reason for this detection failure remains to be explored.

In conclusion, the modified HPLC method is able to detect Trp and Kyn simultaneously in uremic patients, satisfying the requirements for IDO determination in clinical practice.

\section{Acknowledgements}

The authors thank the Medical Research Center (Guangdong General Hospital, Guangzhou, China) core facilities for assistance and Tie-feng Chen for technical assistance. The study was supported by a grant from the National Key Technology R\&D Program (no. 2011BAI10B08) and the National Clinical Key Specialty Construction Preparatory Projects.

\section{References}

1. Stenvinkel P, Heimbürger O, Paultre F, Diczfalusy U, Wang T, Berglund L and Jogestrand T: Strong association between malnutrition, inflammation, and atherosclerosis in chronic renal failure. Kidney Int 55: 1899-1911, 1999.

2. Medzhitov R, Shevach EM, Trinchieri G, et al: Highlights of 10 years of immunology in Nature Reviews Immunology. Nat Rev Immunol 11: 693-702, 2011.

3. Mellor AL and Munn DH: IDO expression by dendritic cells: tolerance and tryptophan catabolism. Nat Rev Immunol 4: 762-774, 2004.

4. Eleftheriadis T, Liakopoulos V, Antoniadi G, Stefanidis I and Galaktidou G: Indoleamine 2,3-dioxygenase is increased in hemodialysis patients and affects immune response to hepatitis B vaccination. Vaccine 29: 2242-2247, 2011.

5. Schefold JC,Zeden JP, Fotopoulou C, et al: Increased indoleamine 2,3-dioxygenase (IDO) activity and elevated serum levels of tryptophan catabolites in patients with chronic kidney disease: a possible link between chronic inflammation and uraemic symptoms. Nephrol Dial Transplant 24: 1901-1908, 2009.

6. Eleftheriadis T, Antoniadi G, Liakopoulos V, Stefanidis I and Galaktidou G: Plasma indoleamine 2,3-dioxygenase concentration is increased in hemodialysis patients and may contribute to the pathogenesis of coronary heart disease. Ren Fail 34: 68-72, 2012.

7. Pallotta MT, Orabona C, Volpi C, et al: Indoleamine 2,3-dioxygenase is a signaling protein in long-term tolerance by dendritic cells. Nat Immunol 12: 870-878, 2011.

8. Brandacher G, Cakar F, Winkler C, et al: Non-invasive monitoring of kidney allograft rejection through IDO metabolism evaluation. Kidney Int 71: 60-67, 2007.

9. Pawlak D, Tankiewicz A, Mysliwiec P and Buczko W: Tryptophan metabolism via the kynurenine pathway in experimental chronic renal failure. Nephron 90: 328-335, 2002.

10. Liu L, Chen Y, Zhang Y, Wang F and Chen Z: Determination of tryptophan and kynurenine in human plasma by liquid chromatography-electrochemical detection with multi-wall carbon nanotube-modified glassy carbon electrode. Biomed Chromatogr 25: 938-942, 2011.

11. Wang R and Tang A: Simultaneous determination of kynurenine and tryptophan in serum by high performance liquid chromatography. Se Pu 24: 140-143, 2006 (In Chinese).

12. Zhen Q, Xu B, Ma L, Tian G, Tang X and Ding M: Simultaneous determination of tryptophan, kynurenine and 5-hydroxytryptamine by HPLC: Application in uremic patients undergoing hemodialysis. Clin Biochem 44: 226-230, 2011.

13. Koenig P, Nagl C, Neurauter G, Schennach H, Brandacher G and Fuchs D: Enhanced degradation of tryptophan in patients on hemodialysis. Clin Nephrol 74: 465-470, 2010. 